in a rabbit model, ${ }^{7}$ we did not encounter side effects in our patient. The favourable outcome of the case presented suggests that topical amikacin should be considered an effective and safe drug for the treatment of Nocardia keratitis.

PETER OLIVER DENK SOLON THANOS HANS-JÜRGEN THIEL

University Eye Clinic, Tübingen, Germany

Correspondence to: Oliver Denk, Schleichstrasse 12, 72076 Tübingen, Germany.

Accepted for publication 28 June 1996

1 Boiron P, Provost F, Chevrier G, Dupont B. Review of nocardial infections in France 1987 Review of nocardial infections in France 19871990. Eur $\mathcal{J}$
11:709-14.

2 Chen CJ. Nocardia asteroides endophthalmitis. Ophthalmic Surg 1983;14:502-5.

3 Dewsnup DH, Wright DN. In vitro susceptibility of Nocardia asteroides to 25 antimicrobial agents. Antimicrob Agents Chemother 1984;25: 165-7

4 Douglas RM, Grove DI, Elliott J, Looke DFM, Jordan AS. Corneal ulceration due to Nocardia Jordan AS. Corneal ulceration due to Nocardia 20.

5 Helm CJ, Holland GN, Lin R, Berlin OGW, Bruckner DA. Comparison of topical antibiotics for treating mycobacterium fortuitum keratitis in an animal model. Am $\mathcal{f}$ Ophthalmol 1993;116: 700-7.

6 Hirst LW, Harrison GK, Merz WG, Stark WJ. Nocardia asteroides keratitis. $\mathrm{Br} \mathcal{F}$ Ophthalmol 1979;63:449-54.

7 Husain N, Matoba AY, Wilhelmus KR, Jones DB. Isolation and therapy of Nocardia keratitis. Invest Ophthalmol Vis Sci 1995;36 (Suppl):S155.

\section{Recurrent systemic B cell lymphoma of the iris}

EDITOR,-Recognised clinical findings of intraocular lymphoma include vitritis, solid retinal pigment epithelial detachments, diffuse retinal and choroidal infiltrates, retinal haemorrhage, perivasculitis, retinal necrosis, and optic neuritis. ${ }^{1}$ Anterior uveitis secondary to posterior segment intraocular lymphoma is recognised, but isolated primary or recurrent iris lymphoma is rare. We report a case of non-Hodgkin's lymphoma involving only the iris.

\section{CASE REPORT}

A 25-year-old white woman reported blurred vision in the right eye for 3 weeks. Eighteen months earlier, she had presented with a right seventh nerve palsy and was found to have a right nasopharyngeal and parapharyngeal mass extending posteriorly to the right carotid sheath with perineural spread. Biopsy revealed diffuse large B cell lymphoma and lumbar puncture was positive for malignant lymphocytes. She was managed with systemic cyclophosphamide, vincristine, and doxorubicin, and intrathecal methotrexate and cytarabine, followed by external beam radiotherapy to the nasopharyngeal area with eye shielding. At completion of therapy, all repea studies including cerebrospinal fluid examination were normal and she remained in remission until presentation 18 months later.

On examination, the visual acuity was $20 / 30$ in the right eye and $20 / 20$ in the left. Intraocular pressures were $16 \mathrm{~mm} \mathrm{Hg}$ in both eyes. The left eye was normal, the right showed central corneal oedema with fine diffuse keratic precipitates and moderate cells and flare in the anterior chamber. The entire righ iris was thickened and a diffuse solid mass was present superiorly (Fig 1). There was noticeable iris heterochromia, with the right iris

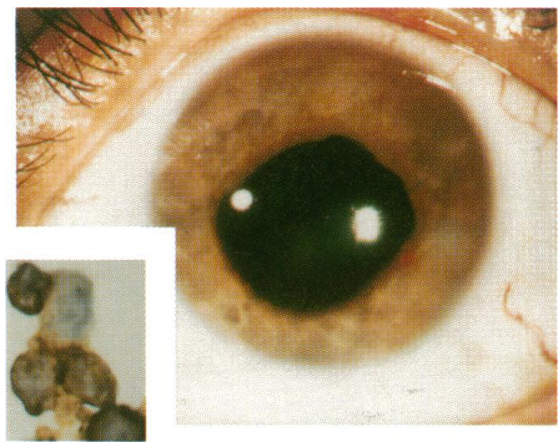

Figure 1 Slit-lamp photograph of the right eye showing diffuse nodular iris thickening. Inset: high power photomicrograph of cytological specimen showing lymphoma cells and positive immunocytochemical staining for the pan B cell marker CD20 (L26).

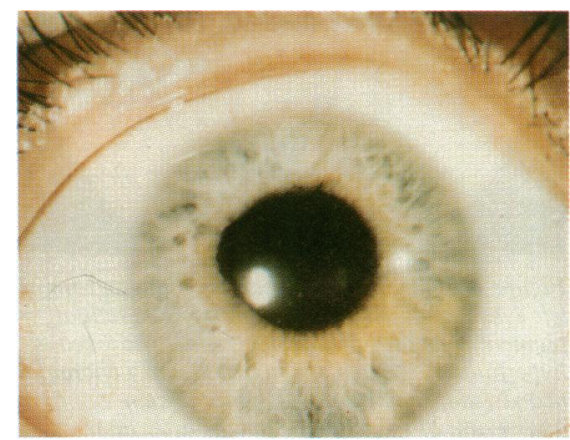

Figure 2 Slit-lamp photograph of the right iris showing iris crypts and mild persistent ectropion uvea 3 months after radiotherapy. There is no residual iris thickening.

brown and the left iris blue. Ectropion uvea was present superiorly, but there was no iris neovascularisation or lens abnormality. The posterior segment examination was unremarkable. Systemic examination was normal.

Uncomplicated fine needle aspiration biopsy of the solid mass was performed via a superior temporal approach. Cytological evaluation of the specimen showed the presence of large atypical lymphocytes, morphologically compatible with non-Hodgkin's lymphoma. Immunocytochemical studies revealed positive staining of the cells for CD20 (L26) (Fig 1: inset), a pan B cell marker, and negative staining for CD45RO (UCHL1), a pan $T$ cell marker. The patient underwent anterior external beam radiotherapy to the right iris, receiving $1980 \mathrm{cGy}$ in 11 equal fractions. Three months later, the visual acuity was 20/20 and there was no thickening of the iris in the right eye, although the iris was less mobile than the left and there was some persistent ectropion uvea of the pupillary margin (Fig 2).

\section{COMMENT}

Anterior segment intraocular lymphoma in the presence of a normal fundus is rare. The first case reported was one in which an iris nodule was confirmed to be a lymphomatous lesion histopathologically after enucleation. To our knowledge, six other cases have been fully reported, ${ }^{2-5}$ two presenting with nongranulomatous uveitis, ${ }^{34}$ one with neovascular glaucoma, ${ }^{2}$ and three with diffuse iris thickening. ${ }^{35}$ In all six cases, as well as in this case, the iris lymphoma was associated with systemic non-Hodgkin's lymphoma, which had been previously diagnosed in five, rather than primary CNS lymphoma. This is not surprising as systemic lymphoma is typically associated with uveal tract ocular involvement, whereas primary CNS lymphoma frequently involves the retina and optic nerve. However, it was unusual that all four of the six cases that reported cell type were $T$ cell lymphoma ${ }^{3}$ since only $5 \%$ of systemic lymphoma is of $T$ cell or mycosis fungoides origin. As more cases of iris lymphoma are categorised accurately by immunofluorescent cell surface marker antigens, and as our case suggests, $B$ cell iris lymphoma may be diagnosed more frequently. The mechanism of involvement of the right iris from the ipsilateral nasopharynx could have included direct extension along the sympathetic chain, CNS spread secondary to perineural involvement, or metastasis.

Three reported patients were treated with systemic chemotherapy, ${ }^{3}{ }^{4}$ two because of simultaneous recurrence of systemic lymphoma, ${ }^{23}$ two were enucleated, ${ }^{35}$ and one was treated with external beam radiotherapy. ${ }^{3}$ One patient treated by systemic chemotherapy had progression of the ocular lesion but the other three cases treated conservatively regressed after therapy.

While combination chemotherapy is indicated for iris lymphoma with systemic involvement, we suggest external beam radiotherapy may be justified in isolated ocular cases. Radiation induced cataract is expected although this may not be a major consideration if life expectancy is short.

Support provided by the Eye Tumor Research Foundation, Philadelphia, PA, USA.

PHILIP G HYKIN

Ocular Oncology Service and the Retina Service, Wills Eye Hospital,

Thomas fefferson University, Philadelphia, PA, USA

JERRY A SHIELDS CAROL L SHIELDS

Ocular Oncology Service, Wills Eye Hospital, Thomas fefferson University, Philadelphia, PA, USA

HORMOZ EHYA

Department of Pathology, Fox Chase Cancer Center, Philadelphia, PA, USA

ELIZABETH SIDERIDES

St foseph Medical Centre, Stamford, CT, USA

Correspondence to: Jerry A Shields, MD, Ocular Oncology Service, Wills Eye Hospital, 900 Walnut Street, Philadelphia, PA 19107, USA.

Accepted for publication 28 June 1996

1 Shields JA, Shields CL. Intraocular tumors: a text and atlas. Philadelphia: WB Saunders, 1992:498-510.

2 Locasio JA. Neoplastic causes of anterior segment inflammation. Int Ophthalmol Clin 1983; 23:111-21

3 Goldey SH, Stern GA, Oblon DJ, Mendenhall NP, Smith LJ, Duque RE. Immunophenotypic characterisation of an unusual T-cell lymphoma presenting as anterior uveitis. Arch Ophthalmol presenting as anterior

4 Hanamura A, Maeda H, Kuwayama W, Takano Y. Infiltration into the aqueous humor in a patient with malignant lymphoma. fap $\mathcal{f}$ Clin Haematol 1992;33:1845-50

5 Jensen OA, Johansen S, Kiss K. Intraocular T-cell lymphoma mimicking intraocular disease. Graefes Arch Clin Exp Ophthalmol 1994;232: 148-52.

\section{Treatment of subfoveal pigment} epithelial detachments with distant extrafoveal choroidal neovascular membranes

EDTTOR,-Subfoveal retinal pigment epithelial detachments larger than 1 disc diameter in patients over the age of 55 have a poor visual prognosis. ${ }^{1}$ Laser photocoagulation of a contiguous extrafoveal choroidal neovascular membrane sometimes causes flattening of a retinal pigment epithelial detachment and 

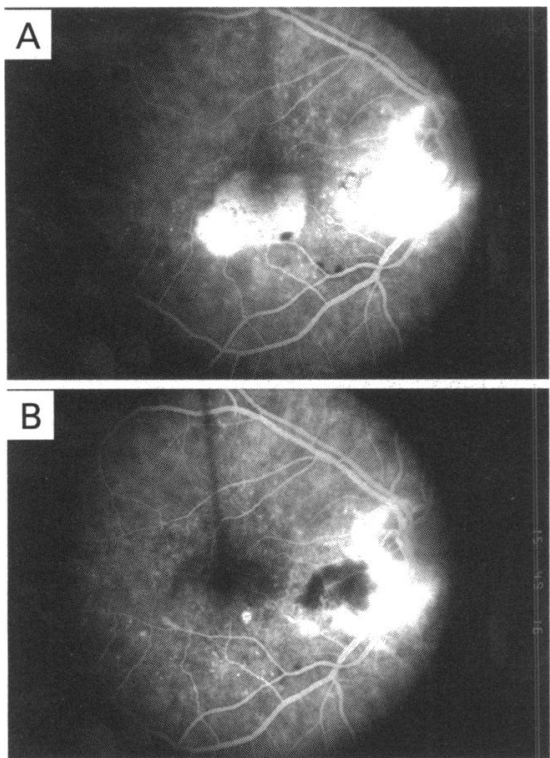

Figure 1 Case 1. (A) Fluorescein angiogram of right eye. Visual acuity was 20/200. There was a 2 disc diameter subfoveal pigment epithelial detachment with smooth borders. The juxtapapillary choroidal neovascular membrane was located inferotemporally adjacent to the optic nerve. There was a distance of less than 1 disc diameter between the edge of the choroidal neovascular membrane and the edge of the retinal pigment epithelial detachment. (B) Fluorescein angiogram of the same eye, 5 weeks after treatment. Visual acuity was 20/20 and the retinal pigment epithelial detachment had resolved.

improvement of visual acuity. ${ }^{23}$ We successfully treated two patients who had serous subfoveal pigment epithelial detachments and juxtapapillary choroidal neovascular membranes. The juxtapapillary choroidal neovascular membranes were extrafoveal and were not contiguous with the retinal pigment epithelial detachments. In addition, the areas of laser photocoagulation were not contiguous with the retinal pigment epithelial detachments.

CASE 1

A 73-year-old woman presented with decreased vision in her right eye for 1 week. Visual acuity was 20/200 right eye, 20/25 left eye. She had subretinal fluid, haemorrhage, and exudate on the temporal margin of the optic disc and a subfoveal pigment epithelial detachment in her right eye. Fluorescein angiography demonstrated a juxtapapillary choroidal neovascular membrane and a 2 disc diameter subfoveal pigment epithelial detachment in her right eye (Fig 1A).

The juxtapapillary choroidal neovascular membrane was treated with the argon green laser. Three weeks after treatment, the retinal pigment epithelial detachment was flat and the patient's visual acuity was $20 / 20$ (Fig 1B) At her last examination, 2 years after laser treatment, her visual acuity was $20 / 20$. She had no signs of recurrence.

\section{CASE 2}

An 82-year-old woman noted decreased visual acuity in her left eye for 2 months. Her visual acuity was $20 / 40$ right eye and 20/200 left eye. She had subretinal fluid, haemorrhage, and exudate on the temporal margin of the optic disc and a subfoveal pigment epithelial detachment in her left eye. A fluorescein angiogram showed a juxtapapillary choroidal neovascular membrane and a subfoveal pig-
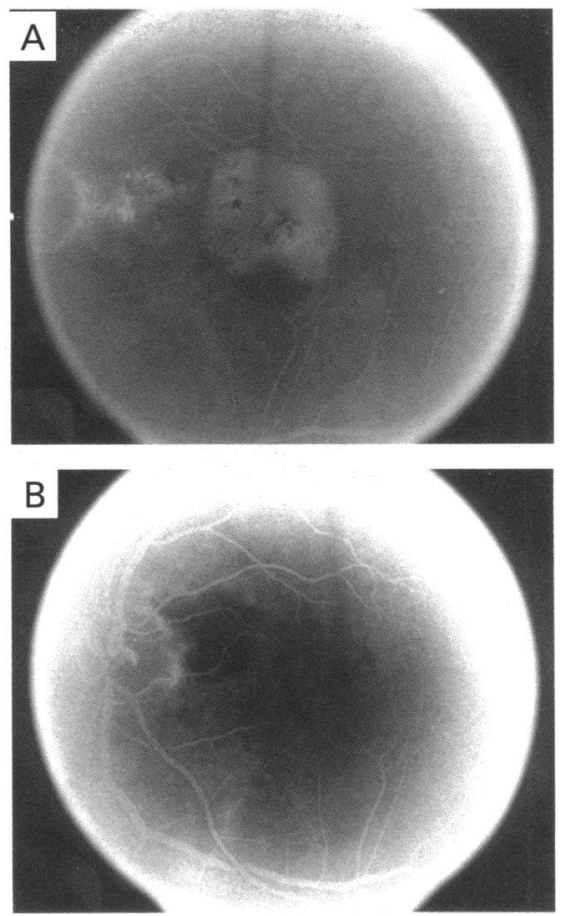

Figure 2 Case 2. (A) Fluorescein angiogram of left eye. Visual acuity was 20/200. The retinal pigment epithelial detachment under the fovea is hyperfluorescent as is the juxtapapillary choroidal neovascular membrane. (B) Fluorescein angiogram of the left eye 3 weeks after laser photocoagulation. Visual acuity was $20 / 20$ and the retinal pigment epithelial detachment had resolved.

ment epithelial detachment in her left eye (Fig 2A) The juxtapapillary choroidal neovascular membrane was treated with argon green laser. Three weeks later, the retinal pigment epithelial detachment was flat, and her visual acuity was 20/50 (Fig 2B) Her last examination was 2 years after the laser treatment. Her visual acuity was 20/25. She had no signs of recurrence.

\section{COMMENT}

The juxtapapillary choroidal neovascular membranes in these two patients were clinically and angiographically distant from the subfoveal serous retinal pigment epithelial detachments. There are three possible ways that laser photocoagulation of a juxtapapillary choroidal neovascular membrane could cause flattening of a distant subfoveal pigment epithelial detachment. Firstly, and most likely, the choroidal neovascular membrane may have been leaking fluid under the nondetached retinal pigment epithelium with enough force to cause a distant retinal pigment epithelial detachment. Photocoagulation of the choroidal neovascular membrane could ablate the source of the subretinal pigment epithelial fluid and cause rapid resolution of the subfoveal pigment epithelial detachment. Secondly, there may have been an occult connection between the choroidal neovascular membrane and the retinal pigment epithelial detachment that was not detectable angiographically. The feeder vessels to such an occult area of choroidal neovascular membrane may have been obliterated by the laser treatment causing the retinal pigment epithelial detachment to flatten. Finally, the retinal pigment epithelial detachment may have arisen independent of the choroidal neovascular membrane. The area of the laser scar may have allowed egress of sufficient fluid from the subretinal pigment epithe- lial space into the subretinal or choroidal space for the pigment epithelial detachment to flatten.

We recommend laser photocoagulation in patients with extrafoveal choroidal neovascular membranes and central visual acuity loss from subfoveal retinal pigment epithelial detachments even if the two lesions are neither clinically nor angiographically connected.

STEVEN M COHEN W SANDERSON GRIZZARD

Retina Associates of Florida, Tampa, Florida,USA

Correspondence to: $\operatorname{Dr}$ Steven M Cohen, 617 Lakeview Road, Suite B, Clearwater, FL 34616, USA.

Accepted for publication 24 May 1996

1 Meredith TA, Bradley RE, Aaberg TM. Natura history of serous detachments of the retinal pigment epithelium. $A m$ f Ophthalmol 1979;88:643-51.

2 Gass JDM. Serous retinal pigment epithelial detachment with a notch: a sign of occult detachment with a notch: a sign of occult 205-20.

3 Maguire JI, Benson WE, Brown GC. Treatmen of foveal pigment epithelial detachments with contiguous extrafoveal choroidal neovascular membranes. Am f Ophthalmol 1990;109:523-9.

Linear scleroderma with orbital involvement: follow up and magnetic resonance imaging

EDITOR,-Scleroderma is a chronic connective tissue disease of unknown aetiology. ${ }^{1}$ Linear scleroderma is an uncommon dermatological disorder which produces an unilateral and localised band-like linear atrophy of the skin and its underlying structures.

Orbital involvement has been described, but is very rare. ${ }^{12}$

CASE REPORT

A 21-year-old woman first presented to an ophthalmologist because of a progressive narrowing of the right palpebral fissure over a period of 3 years with loss of lashes of the eyelids. Further ophthalmic examination was normal. An orbital computed tomogram (CT) was normal.

Three years later there was additional enophthalmia and an entropion of the lower right eyelid was seen (Fig 1). Visual acuity was $8 / 10$ in the right eye and $10 / 10$ in the left Visual field examination and colour vision were normal.

With the exception of a localised area of parietotemporal alopecia on the right side, no other skin defects could be detected. General examination revealed no muscular pain, no arthralgia, no fatigue, and no other systemic symptoms. Laboratory examination of muscle enzymes, tissue antibodies and complement

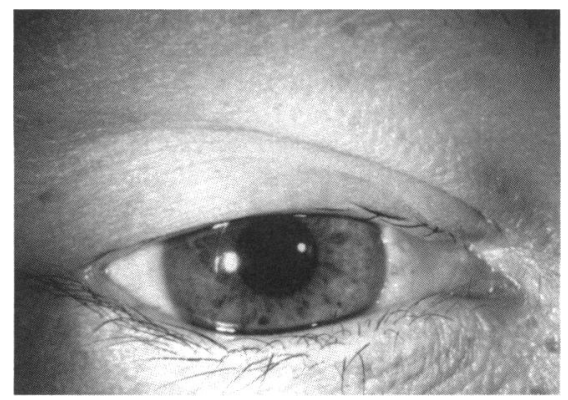

Figure 1 Right eye: note the narrowing of the palpebral fissure and the loss of lashes of the eyelids. 